\title{
Erratum zu: "We have to restructure the way the educational system works from scratch."
}

Abimbola Odugbesan, Edward Stanley Steve und Leslie Carmel Gauditz

\section{Erratum zu:}

Kapitel 8 in: M. E. Kaufmann et al. (Hrsg.), Forschen und Arbeiten im Kontext von Flucht, https://doi.org/10.1007/978-3-658-28380-3_8

Der Name des Autors [Edward Stanley Steve] fehlte auf unserer SpringerLink Website. Dies wurde nun aktualisiert. 